\title{
ABKÜRZUNGEN UND SIGEL
}

\section{häufig zitierter Periodica und Einzelveröffentlichungen}

AA. Archäologischer Anzeiger

AAA. s. AnnLiv

AaA. Art and Archaeology

AAD. Architettura e Arti Decorative

AägArch. Archiv für ägyptische Archäologie

AAel. Archaeologia Aeliana

AAls. Archives Alsaciennes d'Histoire de l'Art

AASOR. Annual of the American Schools of Oriental Research in Jerusalem

AbhBerl. Abhandlungen der Deutschen (PreuBischen) Akademie der Wissenschaften zu Berlin. Phil.-hist. Klasse

AbhHeid. Abhandlungen der Heidelberger AKademie der Wissenschaften. Phil.-hist. Klasse

AbhLeipz. Abhandiungen der (Königlich) Sächsischen Gesellschaft der Wissenschaften. Phil.hist. Klasse

AbhMünch. Abhandlungen der (Königlich) Bayerischen Akademie der Wissenschaften. Hist. Klasse

ACambr. Archaeologia Cambrensis

ACant. Archaeologia Cantiana

ActaArch. Acta Archaeologica

ActaOr. Acta Orientalia

ACuerpFac. Anuario del Cuerpo Facultativo de Archiveros, Bibliotecarios y Arqueólogos

AD. Antike Denkmäler

AdI. Annali dell'Instituto di Corrispondenza Archeologica

AEg. Ancient Egypt

AegForsch. Aegyptologische Forschungen

ÄgM. Mitteilungen des Deutschen Instituts für Ägyptische Altertumskunde in Kairo

ÄgZ. Zeitschrift für ägyptische Sprache und Altertumskunde

AEM. Archaeologisch-Epigraphische Mitteilungen aus Oesterreich

AeR. Atene e Roma

AEsp. Archivo Español de Arte y Arqueologia Äz. s. ÄgZ.

AfO. s. AOF.
Afrit. Africa Italiana

AJ. s. Ant J.

AJA. American Journal of Archaeology

A JPh. American Journal of Philology

AIPhOr. Annuaire de l'Institut de Philologie et d'Histoire Orientales et Slaves

A JSL. American Journal of Semitic Languages and Literatures

AK. Archiv für Keilschriftforschung

Albizzati, Vas. del Vat. C. Albizzati, Vasi Antichi Dipinti del Vaticano

AM. Mitteilungen des Deutschen Archäologischen Instituts. Athenische Abteilung

Amelung, Antiken in Florenz. W. Amelung, Führer durch die Antiken in Florenz.

Amelung, Vat. Kat. W. Amelung, Die Sculpturen des Vaticanischen Museums

AmtlBer. Amtliche Berichte aus den Königlichen Kunstsammlungen

AnnArchBrux. Annales de la Société Royale d'Archéologie de Bruxelles

AnnInst. Annales Institutorum

AnnLiv. Annals of Archaeology and Anthropology (University of Liverpool)

AnnPisa Annali della Reale Scuola Normale Superiore di Pisa. Sezione di Lettere

AnnPlovdiv Annuaire de la Bibliothèque Nationale et Musée de Plovdiv

AntCl. L'Antiquité Classique

AntJ. The Antiquaries Journal

AnzAW. Anzeiger für die Altertumswissenschaft AnzSchwAlt. Anzeiger für Schweizerische Altertumskunde

AnzWien Anzeiger der Akademie der Wissenschaften. Wien. Phil,-hist. Klasse

AO. Der Alte Orient

AOF. Archiv für Orientforschung

AOr. Archiv Orientálni

APF. Archiv für Papyrusforschung

ArchClass. Archeologia Classica

ArchDalm. Archivio Storico per le Dalmazia 
ArchErt. Archaeologiai Ertesitö

Arch J. The Archaeological Journal

ArndtBr. Griechische und Römische Porträts.

(Hrsg. von P. Arndt - F. Bruckmann)

ArtB. The Art Bulletin

ARW. Archiv für Religionswissenschaft

ASA. S. AnzSchwAlt.

ASAntEg. Annales du Service des Antiquités de l'Égypte

ASAtene Annuario della Reale Scuola Archeologica di Atene

AttiAccPont. Atti della Pontificia Accademia Romana di Archeologia

AttiAccTorino Atti della Reale Accademia delle Scienze di Torino

Atti ... CStCol. Atti del ... Congresso di Studi Coloniali

Atti ... CStR. Atti del ... Congresso Nazionale di Studi Romani

AttiIstVen. Atti dell' Istituto Veneto di Scienze, Lettere ed Arti

AttiMGrecia Atti e Memorie della Società Magna Grecia

AttiProgrSc. Atti della Società Italiana per il Progresso delle Scienze

AttiSocIstr. Atti e Memorie della Società Istriana di Archeologia e Storia Patria

AuA. Antike und Abendland. Hrsg. von B. Snell

AuChr. Antike und Christentum

AV. ersetzt durch Gerhard, AV.

AvP. Altertümer von Pergamon

AZ. Archäologische Zeitung

BA. Bulletin Archéologique du Comité des Travaux Historiques et Archéologiques

Babelon, Camées de la Bibl. Nat. E. Babelon, Catalogue des Camées Antiques et Modernes de la Bibliothèque Nationale

Babelon-Blanchet E. Babelon - J.-A. Blanchet, Catalogue des Bronzes Antiques de la Bibliothèque Nationale

BAC. Bullettino di Archeologia Cristiana

BAcPol. Bulletin International de l'Académie Polonaise des Sciences et des Lettres. Classe de Philologie

BadFB. Badische Fundberichte

BAntBeschav. Bulletin van de Vereeniging tot Bevordering der Kennis van de Antike Beschaving

BAntFr. Bulletin de la Société Nationale des Antiquaires de France

BArchAlex. Bulletin de la Société Archéologique d'Alexandrie

BASOR. Bulletin of the American Schools of Oriental Research in Jerusalem

BASPR. Bulletin of the American School of Prehistoric Research

BayBll. Bayerische Blätter für das GymnasialSchulwesen
BayVBll. Bayerische Vorgeschichtsblätter

BBudé Bulletin de l'Association Guillaume Budé BBudéSCr. Bulletin de l'Association Guillaume

Budé. Supplément Critique

$\mathrm{BCH}$. Bulletin de Correspondance Hellénique

BCMI. Buletinul Comisiunii Monumentelor Istorice Bd'A. Bollettino d'Arte

BDetrI. Bulletin of the Detroit Institute of Arts of the City of Detroit

BdI. Bullettino dell'Instituto di Corrispondenza Archeologica

Beazley, AV. J. D. Beazley, Attische Vasenmaler des rotfigurigen Stils

Beazley, ARV. s. Beazley, AVP.

Beazley, AVP. J. D. Beazley, Attic Red-Figure Vase-Painters

Beazley-Magi J. D. Beazley - F. Magi, La Raccolta Benedetto Guglielmi nel Museo Gregoriano Etrusco

Benndorf-Schöne O. Benndorf - R. Schöne, Die antiken Bildwerke des Lateranensischen $\mathrm{Mu}$ seums beschrieben

BerlMus. Berliner Museen. Berichte aus den Preußischen Kunstsammlungen

BerRGK. Deutsches Archäol. Institut. Bericht der Römisch-Germanischen Kommission

Beschr. ant. Skulpt. Berlin Kurze Beschreibung der antiken Skulpturen im Alten Museum

BFil. Bollettino di Filologia Classica

BIA. Bollettino dal Reale Istituto di Archeologia e Storia dell'Arte

$\mathrm{BJb}$. ersetzt durch $\mathrm{BJbb}$.

$\mathrm{BJbb}$. Bonner Jahrbücher

BiblR. The Biblical Review

BIBulg. Bulletin de l'Institut Archéologique Bulgare

BIE. Bulletin de l'Institut Égyptien

Bieber, Kat. Cassel M. Bieber, Die antiken Skulpturen und Bronzen des Königl. Museum Fridericianum in Cassel

BIFAO. Bulletin de l'Institut Français d'Archéologie Orientale

BII. Bulletin of the Iranian Institut

Billedtavler Ny Carlsberg Glyptotek. Billedtavler til Kataloget over Antike Kunstvaerker BiOr. Bibliotheca Orientalis

BllMfr. Blätter für Münzfreunde

Blümel, Kat. Berlin, Röm. Bildn. Staatl. Museen zu Berlin. Katalog der Sammlung antiker Skulpturen. C. Blümel, Römische Bildnisse

Blümel, Kat. Berlin, Röm. Kop. 4. Jh. Staatl. Museen zu Berlin. Katalog der Sammlung antiker Skulpturen V. C. Blümel, Römische Kopien griechischer Skulpturen des IV. Jahrhunderts v. Chr.

Bl ümel, Kat. Berlin, Röm. Kopien 5. Jh. Staatı. Museen zu Berlin. Katalog der Sammlung 
antiker Skulpturen IV. C. Blümel, Römische Kopien griechischer Skulpturen des V. Jahrhunderts v. Chr.

Blümel, Kat. Berlin, Skulpt. 6. u. 5. Jh. Staatl. Museen zu Berlin. Katalog der Sammlung antiker Skulpturen II I. C. Blümel, Griechische Skulpturen des VI. und V. Jahrhunderts v. Chr.

Blümel, Kat. Berlin, Skulpt. 5. u. 4. Jh. Staatl Museen zu Berlin. Katalog der Sammlung antiker Skulpturen III. C. Blümel, Die griechischen Skulpturen des V. und IV. Jahrhunderts v. Chr.

Blümner, Technologie H. Blumner, Technologie und Terminologie der Gewerbe und Künste bei Griechen und Römern

BMC. A Catalogue of the Greek Coins in the British Museum

BMetrMus. Bulletin of the Metropolitan Museum of Art

BMImpR. Bullettino del Museo dell'Impero Romano

BMon. Bulletin Monumental

BMusArt Bulletin des Musées Royaux d'Art et d'Histoire

BMusBeyr. Bulletin du Musée de Beyrouth

BMusFA. Museum of Fine Arts Bulletin

BMusFr. Bulletin des Musées de France

BNJ. Byzantinisch-Neugriechische Jahrbücher

BOffInt. Bulletin de l'Office International des Instituts d'Archéologie et d'Histoire de l'Art

BollStM. Bollettino dell'Associazione Internationale degli Studi Mediterranei

BOran Bulletin 1 rimestriel des Antiquités Africaines Recueillies par les Soins de la Socété de Géographie et d'Archéologie de la Province d'Oran

BphW. Berliner philologische Wochenschrift

BPI. Bullettino di Paletnolugia Italiana

BrBr. Brunn-Bruckmanns Denkmäler griechischer und römischer Skulptur

Brit. Mus. Cat., Hinks, Paintings R. P. Hinks, Catalogue of the Greek, Etruscan and Roman Paintings and Mosaics in the British Museum

Brit. Mus. Cat., Marshall, Finger Rings F. H. Marshall, Catalogue of the Finger Rings, Greek, Etruscan and Roman in the ... British Museum

Brit. Mus. Cat., Marshall, Jewellery F. H. Marshall, Catalogue of the Jewellery, Greek, Etruscan and Roman in the British Museum

Brit. Mus. Cat., Smith, Sculpture A. H. Smith, A Catalogue of Sculpture in the Department of Greek and Roman Antiquities, British Museum

Brit. Mus. Cat., Vases Catalogue of the Greek and Etruscan Vases in the British Museum

Brit. Mus. Cat., Walters, Bronzes H. B. Walters, Catalogue of the Bronzes, Greek, Roman and Etruscan in the British Museum
Brit. Mus. Cat., Walters, Gems H. B. Walters. Catalogue of the Engraved Gems and Cameos, Greek, Etruscan and Roman in the British Museurn

Brit. Mus. Cat., Walters, Lamps H. B. Walters, Catalogue of the Greek and Roman Lamps in the British Museum

Brit. Mus. Cat., IValters, Silver Plate H. B. Walters, Catalogue of the Silver Plate (Greek, Etruscan and Roman) in the British Museum

Brit. Mus. Cat., Walters, Terracottas H. B. Walters, Catalogue of the Terracottas in the British Museum

BrMQu. The British Museum Quarterly BrooklMQu. The Brooklyn Museum Quarterly BSA. Annual of the British School at Athens

BSocBulg. Bulletin de la Société Archéologique Bulgare

BSOSt. Bulletin of the School of Oriental Studies

BSR. Papers of the British School at Rome

BullCom. Bullettino della Commissione Archeologica Comunale di Roma

BullGov. Bullettino della Commissione Archeologica del Governatorato di Roma

Bulle, SchM. H. Bulle, Der Schöne Mensch im Altertum

BullNap. Bollettino Archeologico Napolitano

BurlMag. Burlington Magazine for Connoisseurs

Burs. Jahresbericht über die Fortschritte der klassischen Altertumswissenschaft (hrsg. von C. Bursian)

BWPr. Winckelmannsprogramm der Archäologischen Gesellschaft zu Berlin

BYaleU. Bulletin of the Associates in Fine Arts at Yale University

ByzZ. Byzantinische Zeitschrift

$B Z$. ersetzt durch ByzZ.

CAH. The Cambridge Ancient History

CArch. Cahiers Archéologiques

CArchAls. Cahiers d'Archéologie et d'Histoire d'Alsace

Caskey, Cat. Sculpt. Boston Boston, Museum of Fine Arts. L. D. Caskey, Catalogue of Greek and Roman Sculpture

Casson, Cat. Acrop. Mus. Catalogue of the Acropolis-Museum II. S. Casson, Sculpture and Architectural Fragments. With a Section upon the Terracottas by D. Brooke.

Cd'A. Cahiers d'Art

CHistArch. Cahiers d'Histoire et d'Archéologie ChrAM. Chronika Archeologii ta Mistectwa

ChrEg. Chronique d'Égypte

CIA. Corpus Inscriptionum Atticarum

CIE. Corpus Inscriptionum Etruscarum

CIG. Corpus Inscriptionum Graecarum

CIL. Corpus Inscriptionum Latinarum 
CIS. Corpus Inscriptionum Semiticarum

Clarac F. de Clarac, Musée de Sculpture Antique et Moderne

ClB. The Classical Bulletin

CIJ. The Classical Journal

CIPh. Classical Philology

ClQu. The Classical Qarterly

CIRev. The Classical Review

ClW. The Classical Weekly

Collignon-Couve M. Collignon - L. Couve, Catalogue des Vases Peints du Musée National d'Athènes

CongrIntOr. Actes du $20^{\circ}$ Congrès International des Orientalistes

CongrStorArch. Atti del ... Congresso Nazionale di Storia dell'Architettura

ConvStorArch. Atti del ... Convegno Nazionale di Storia dell'Architettura

Conze, Att. Grabreliefs Die attischen Grabreliefs. Hrsg. von A. Conze

Corolla Curtius Corolla Ludwig Curtius zum 6o. Geburtstag dargebracht

CRAcInscr. Comtes-Rendus des Séances. Académie des Inscriptions et Belles-Lettres

Crd'A. La Critica d'Arte

CRPétersb. Compte-Rendu de la Commission Impériale Archéologique St. Pétersbourg

Curtius, AK. I Handbuch der KunstwissenschaftDie antike Kunst I. L. Curtius, Ägypten und Vorderasien

Curtius, AK. II Handbuch der Kunstwissenschaft. Die antike Kunst II. L. Curtius, Die klassische Kunst Griechenlands

CVA. Corpus Vasorum Antiquorum

CVind. Commentationes Vindobonenses

DA. Dictionnaire des Antiquités Grecques et Romaines ... sous la Direction de Ch. Darenberg et Edm. Saglio

Dalton, Treas. of Oxus $\quad$ O. M. Dalton, The Treasure of the Oxus with other Objects from Ancient Persia and India, bequeathed to the Trustees of the British Museum by Sir. A. W. Franks

Dawkins, Artemis Orthia The Sanctuary of Artemis Orthia at Sparta. Ed. by R.M. Dawkins

Délos Exploration Archéologique de Délos faite par l'École Française d'Athènes

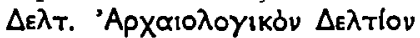

DJbNum. Deutsches Jahrbuch für Numismatik Dittenberger, Sylloge W. Dittenberger, Sylloge Inscriptionum Graecarum

DLZ. Deutsche Literaturzeitung

DMBll. Deutsche Münzblätter

Dokl. Doklady Rossijskoj Akademii Nauk

Dolg. Dolgozatok a M. Kir. Ferencz József -Tudományegyetem és a M.Kir. Horthy Miklós-Tudom nyegyetem régiségtudományi intézetéböl
DTCFD, Ankara Universitesi, Dil ve TarihCoğrafya Fakültesi Dergisi

Dütschke H. Dütschke, Antike Bildwerke in Oberitalien

Dura-Europos, Fin. Rep. The Excavations at Dura-Europos. Final Report

Dura-Europos, Prel. Rep. The Excavations at Dura-Europos. Preliminary Report

EA. Photographische Einzelaufnahmen antiker Skulpturen (,Arndt-Amelung ")

EE. ersetzt durch EphEp.

EgRel. Egyptian Religion

Eichler-Kris F. Eichler - E. Kris, Die Kameen im Kunsthistorischen Museum (Wien)

Enc. Phot. TEL Encyclopédie Photographique de l'Art. Éditions TEL

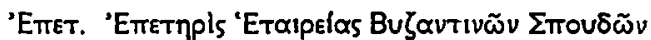

EphEp. Ephemeris Epigraphica

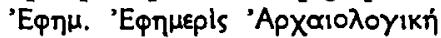

ESA. Eurasia Septentrionalis Antiqua

Espér. E. Espérandieu, Recueil Général đes Bas-Reliefs, Statues et Bustes de la Gaule Romaine

EtCl. Les Études Classiques

Exped. Sieglin Veröffentlichungen der Ernst von Sieglin Expedition in Aegypten

FA. Fasti Archaeologici

Fairbanks, Cat. Vas. Boston Museum of Fine Arts, Boston. Catalogue of Greek and Etruscan Vases. I A. Fairbanks, Early Vases, preceeding Athenian Black-Figured Ware

FBSchwab. ersetzt durch FBSchwaben

FBSchwaben Fundberichte aus Schwaben

FCA. (Kock) Fragmenta Comicorum Atticorum. Ed. Th. Kock

FCG. (Kaibel) Fragmenta Comicorum Graecorum. Ed. G. Kaibel

FCG. (Meineke) Fragmenta Comicorum Graecorum. Coll. et disposuit A. Meineke

FdD. Fouilles de Delphes

FEG. (Kinkel) Fragmenta Epicorum Graecorum. Disposuit Commentarium Criticum adiecit G. Kinkel

FelRav. Felix Ravenna

FHG. Fragmenta Historicorum Graecorum

FolArch. Folia Archaeologica

FR. A. Furtwängler - K. Reichhold, Griechischo Vasenmalerei

İrMZ. Frankfurter Münzzeitung

Führer Antiqu. Berlin I Bronzen Führer durch das Antiquarium I Bronzen

FuF. Forschungen und Fortschritte

Furtwängler, AG. A. Furtwängler, Die antiken Gemmen

Furtwängler, Geschn. Steine Berlin A. Furtwängler, Beschreibung der geschnittenen Steine im Antiquarium 
Furtwängler, MW. A. Furtwängler, Meisterwerke der griechischen Plastik

Furtwängler, Vasenslg. Berlin A. Furtwängler, Beschreibung der Vasensammlung im Antiquarium

FW., Gipsabgüsse Kgl. Museen zu Berlin. Die Gipsabgüsse antiker Bildwerke in historischer Folge erklärt von C. Friederichs. Neu bearb. von $P$. Wolters

GaR. Greece and Rome

GArb. Geistige Arbeit

GazArch. Gazette Archéologique

GazBA. Gazette des Beaux-Arts

Gercke-Norden A. Gercke-E. Norden, Einleitung in die Altertumswissenschaft

Gerhard, AV. E. Gerhard, Auserlesene Griechische Vasenbilder

Gerhard, ES. E. Gerhard, Etruskische Spiegel

Gerhard, EKV. E. Gerhard, Etruskische und Kampanische Vasentilder des Königl. Museums zu Berlin

Gervasio M. Gervasio, Bronzi Arcaici e Ceramica Geometrica nel Museo di Bari

GGA. Göttingische Gelehrte Anzeigen

GIHrvM. Glasnik Hrvatskih Zemalskih Muzeja u Sarajevu

GlMBosn. Glasnik Zemalskog Muzeja u Bosni i Hercegovini

GIMSlov. Glasnik Muzejskega Društva za Slovenijo

GodNarM. Godišnik na Narodnija Muzej

GodPlovdiv Godišnik na Narodnija Archeolog. Musei Plovdiv

Graef-Langlotz Die antiken Vasen von der Akropolis zu Athen. Hrsg. von B. Graef (später) und E. Langlotz

Guida Ruesch A. Ruesch, Guida Illustrata del Museo Nazionale di Napoli

Gymn. Das Gymnasium

HallWPr. Hallisches Winckelmannsprogramm

HarvSt. Harvard Studies in Classical Philology

HAW. Handbuch der (klassischen) Altertumswissenschaft. Begründet von Iwan Müller, hrsg. von W. Otto

HBr. P. Herrmann, Denkmäler der Malerei des Altertums (Bruckmann)

HdArch. Handbuch der Archäologie im Rahmen des Handbuchs der Altertumswissenschaft. Hrsg. von W. Otto, fortgeführt von R. Herbig

Helbig W. Helbig, Führer durch die öffentlichen Sammlungen klassischer Altertümer in Kom

Helbig, Wgm. W. Helbig, Wandgemälde der vom Vesuv verschütteten Städte Campaniens

Herrmann, Skulpt.-Slg. Dresden P. Herrmann, Verzeichnis der antiken Originalwerke der Staatl. Skulpturensammlung zu Dresden
Heuzey, Cat. Terr. Cuit. Louvre L. Heuzey, Catalogue des Figurines Antiques de Terre Cuite du Musée du Louvre

Heydemann, Vasenslgg. Neapel H. Heydemann, Die Vasensammlungen des Museo Nazionale zu Neapel

Hoppin, Hb. Bf. J. C. Hoppin, A Handbook of Greek Black-Figured Vases with a Chapter on the Red Figured Southern Italian Vases

Hübner, Bildwerke in Madrid E. Hübner, Die antiken Bildwerke in Madrid

HumG. Das Humanistische Gymnasium

HZ. Historische Zeitschrift

JA. s. JAsiat.

Jahn, Vasenslg. München O. Jahn, Beschreibung der Vasensammlung König Ludwigs in der Pinakothek zu München

JAOS. Journal of the American Oriental Society JAsiat. Journal Asiatique

JberEOL. Jaarbericht van het Voor-AziatischEgyptische Gezelschap 'Ex Oriente Lux'

JbKSWien Jahrbuch der Kunsthistorischen Sammlungen in Wien

JBritArchAss. The Journal of the British Archaeological Association

JdI. Jahrbuch des Deutschen Archäologischen Instituts

JEA. The Journal of Egyptian Archaeology

JEMA. The Journal of the Egyptian Medical Association

JEOL. s. JberEOL

IF. Indogermanische Forschungen

IG. Inscriptiones Graecae

IGA. Inscriptiones Graecae Antiquissimae

JHS. Journal of Hellenic Studies

Iliffe (Robinson-Harcum), Cat. Vas. Toronto D. M. Robinson - C. G.Harcum, A Catalogue of the Greek Vases in the Royal Ontario Museum of Archaeology, Toronto. Ed. by J. H. Iliffe

IIIIt. L'Illustrazione Italiana

IllVat. L'Illustrazione Vaticana

ILN. Illustrated London News

JManch. Journal of the Manchester (Egyptian and) Oriental Society

JNES. Journal of Near Eastern Studies

IntArchEthn. Internationales Archiv für Ethnographie

IntCongrArchCrist. Atti del ${ }_{4}$ Congresso Internazionale di Archeologia Cristiana

IntCongrIr. $3^{e}$ Congrès International d'Art et d'Archéologie Iraniens. Mémoires

IntNumCongr. Transactions of the International Numismatic Congress 1936

JPalOrS. The Journal of the Palestine Oriental Society

JPrKS. Jahrbuch der Preußischen Kunstsammlungen 
JRAS. The Journal of the Royal Asiatic Society of Great Britain and Ireland

JRS. The Journal of Roman Studies

JSav. Journal des Savants

JSOR. Journal of the Society of Oriental Research

IyP. Investigación y Progreso

IzvMatKult. Izvestija Gosudarstvennoj Akademii Material'noj Kultury

Kaschnitz-Weinberg, Mag. Vat. G. Kaschnitz-

Weinberg, Sculture del Magazzino del Museo Vaticano

KfA. Die Kunst für Alle

$\mathrm{KiB}$. ersetzt durch Winter, KiB.

Közl. Egyetemes Philologiai Közlöny

KorrBI. Korrespondenzblatt des Gesamtvereines der deutschen Geschichts- und Altertumsvereine

KritB. Kritische Berichte zur kunstgeschichtlichen Literatur

KuARdsch. Kunst- und Antiquitäten-Rundschau

KuK. Kunst und Künstler

KW. Der Kunstwanderer

KwKl. Kwartalnik Klasyczny

Langlotz, Bildhauersch. E. Langlotz, Frühgriechische Bildhauerschulen

Langlotz, Griech. Vas. Würzburg Martin v. Wagner-Museum der Universität Würzburg. E. Langlotz, Griechische Vasen

Lehner, Führer Bonn H. Lehner, Führer durch das Provinzialmuseum in Bonn I. Die antike Abteilung

Lehner, Prov. Mus. Bonn, Abbildungen Das Provinzialmuseum in Bonn. Abbildungen seiner wichtigsten Denkmäler. Hrsg. von $\mathrm{H}$. Lehner

LeipzWBl. Leipziger Winckelmannsblatt

LHum. Lettres d'Humanité

Liddell-Scott H. G. Liddell - R. Scott, A GreekEnglish Lexicon

Listy Listy Filologicke

LZ. Literarisches Zentralblatt

MAGWien Mitteilungen der Anthropologischen Gesellschaft, Wien

MarbJb. Marburger Jahrbuch für Kunstwissenschaft

MarbWPr. Marburger Winckelmann-Programm

Mattingly, Rom. Coins H. Mattingly, Coins of the Roman Empire in the British Museum

Matz-Duhn F.Matz, Antike Bildwerke in Rom mit Ausschluß der größeren Sammlungen. (Weitergef. u. hrsg. von $F$. von Duhn)

MCl. Il Mondo Classico

MD. ersetzt durch Matz-Duhn

MdI. Mitteilungen des Deutschen Archäologischen Instituts

MDOG. Mitteilungen der Deutschen OrientGesellschaft
Meded. Mededeelingen van het Nederl. Historisch Instituut te Rome

Mél. Mélanges d'Archéologie et d'Histoire

MélUnivBeyr. Mélanges de l'Université SaintJoseph, Beyrouth

MemAccLinc. Memorie della Reale Accademia Nazionale dei Lincei

MemAccNapoli Società Reale di Napoli. Memorie della Reale Accademia di Archeologia, Lettere e Belle Arti

MemAccPont. ersetzt durch MemPontAcc.

MémAcInscr. Mémoires présentés par Divers Savants à l'Académie des Inscriptions et Belles-Lettres

MemAmAc. Memoirs of the American Academy in Rome

MemExc. Junta Superior de Excavaciones y Antiguiedades. Memorias

Mem. F. E. R. T. Memorie pubblicate a Cura dell' Istituto Storico-Archeologico F. E. R. T. (di Rodi)

Memoria ersetzt durch MemExc.

MemPontAcc. Atti della Pontificia Accademia Romana di Archeologia. Memorie

Mendel Musées Impériaux Ottomans. G. Mendel, Catalogue des Sculptures Grecques, Romaines et Byzantines

$\checkmark$ Mercklin, Führer Hamburg Führer durch das Hamburgische Museum für Kunst und Gewerbe II. E. v. Mercklin, Griechische und römische Altertümer

MetrMusSt. Metropolitan Museum Studies

MJ. University of Pennsylvania. The Museum Journal

MJb. ersetzt durch MüJb.

MittPhilWien Mitteilungen des Vereines klassischer Philologen in Wien

ML. W. H. Roscher, Ausführliches Lexikon der griechischen und römischen Mythologie

MonAnt. Monumenti Antichi pubblicati per Cura della Reale Accademia dei Lincei

MonInst. Monumenti Inediti pubblicati dall'Instituto Archeologico

MonLinc. s. MonAnt.

MonPiot Fondation Eugène Piot. Monuments et Mémoires

Montfoucon B. de Montfoucon, L'Antiquité Expliquée et Représentée en Figures

MOr. Le Monde Oriental

Ms J. The Museums Journal (London)

MüJb. Münchner Jahrbuch der bildenden Kunst

Müller-Wieseler C. O. Müller - F. Wieseler, Antike Denkmäler zur griechischen Götterlehre

MusHelv. Museum Helveticum

MVAeG. Mitteilungen der Vorderasiatisch-Ägyptischen Gesellschaft 
MVEOL. Mededeelingen en Verhandelingen van het Vooraziatisch-Egyptische Gezelschap 'Ex Oriente Lux'

Myres, Cesnola Coll. The Metropolitan Museum of Art. J. L. Myres, Handbook of the Cesnola Collection of Antiquities from Cyprus

NBArchCrist. Nuovo Bulletino di Archeologia Cristiana

NBdA. Das Neue Bild der Antike. Hrsg. von H. Berve

NdtV. Nachrichtenblatt für deutsche Vorzeit

Neugebauer, Kat. Berlin, Bronzen I Staatl. Museen zu Berlin. Katalog der statuarischen Bronzen im Antiquarium I. K. A. Neugebauer, Die minoischen und archaisch griechischen Bronzen

Neugebauer, Kat. Berlin, Bronzen II Staatl. Museen zu Berlin. Katalog der statuarischen Bronzen im Antiquarium II. K. A. Neugebauer, Die griechischen Bronzen der klassischen Zeit und des Hellenismus

Neugebauer, Vasen Führer durch das Antiquarium II. K. A. Neugebauer, Vasen

$\mathrm{NeV}$. Nova et Vetera

NGG. Nachrichten von der Akademie der Wissenschaften in Göttingen

$\mathrm{NJb}$. ersetzt durch $\mathrm{NJbb}$.

NJbb. Neue Jahrbücher für Antike und deutsche Bildung

Nicole, Vas. d'Athènes G. Nicole, Catalogue des Vases Peints du Musée National d'Athènes

Notiziario Ministero delle Colonie. Notiziario Archeologico

NRhH. Nachrichtenblatt für Rheinische Heimatpflege

NSc. Notizie degli Scavi di Antichità

NumChron. The Numismatic Chronicle and Journal of the Numismatic Society

NumCirc. Spink \& Son's Numismatic Circular

NumLBl. Numismatisches Literaturblatt

NumZ. Numismatische Zeitschrift

NZ. ersetzt durch NumZ.

ÖJh. Jahreshefte des Österreichischen Archäologischen Instituts in Wien

OIC. Oriental Institute Communications (Chicago)

OIP. Oriental Institute Publications (Chicago)

Ol.Forsch. Olympische Forschungen. Hrsg. von E. Kunze

OlympiaErg. Olympia. Die Ergebnisse der von dem Deutschen Reich veranstalteten Ausgrabung ... hrsg. von E. Curtius und F. Adler

Olympia, Ubersicht Die Ausgrabungen zu Olympia. Ubersicht der Arbeiten und Funde. Hrsg. von E. Curtius, F. Adler, G. Hirschfeld (später und andere)
Ol.Ber. Bericht über die Ausgrabungen in Olympia

Olynth Excavations at Olynthus

OLZ. Orientalistische Literaturzeitung

OpArch. Skrifter utgivna av Svenska Institutet i Rom. Opuscula Archaeologica

Otto, HdbchArch. ersetzt durch HdArch.

OudhMeded. Oudheidkundige Mededeelingen uit's Rijksmuseum van Oudheden te Leiden

Overbeck, Kunstmythologie J. Overbeck, Griechische Kunstmythologie

Overbeck, Schriftquellen J. Overbeck, Die antiken Schriftquellen zur Geschichte der bildenden Künste bei den Griechen

Ox. Pap. The Oxyrhynchus Papyri. Ed. by B. P. Grenfell - A. S. Hunt

PalExplQu. Palestine Exploration Quarterly PalJb. Palästinajahrbuch

Paribeni, Mus. d. Terme R. Paribeni, Le Terme di Diocleziano e il Museo Nazionale Romano

PASAthens Papers of the American School of Classical Studies at Athens

Perrot-Chipiez G. Perrot - Ch. Chipiez, Histoire de l'Art dans l'Antiquité

Pfuhl, MuZ. E. Pfuhl, Malerei und Zeichnung der Griechen

PhQu. Philological Quarterly

PhW. Philologische Wochenschrift

PP. La Parola del Passato

Pottier, Vas. Louvre E. Pottier, Vases Antiques du Louvre

Poulsen, Kat. Skulpt. Ny Carlsberg Glyptotek. F. Poulsen, Katalog over Antike Skulpturer

Platner-Ashby S. B.Platner-'Th. Ashby, A Topographical Dictionary of Ancient Rome

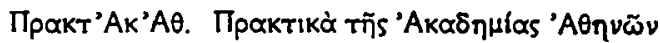

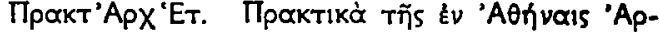

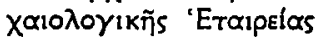

Preller-Robert L. Preller, Griechische Mythologie. 4. Aufl. bearb. von C. Robert

Probl. Problemy Istorii Dokapitalističeskich Obščestv

ProcNewc. Proceedings of the Society of Antiquaries of Newcastle-upon-Tyne

PrKl. Przegląd Klasyczny

PrzArch. Przegląd Archeologiczny

PZ. Prähistorische Zeitschrift

QuAntPal. The Quarterly of the Department of Antiquities in Palestine

QuStat. Quarterly Statement. Palestine Exploration Fund

RA. s. RAssyr.

RA. Revue Archéologique

RAC. Reallexikon für Antike und Christentum

RAComo Rivista Archeologica della Provincia di Como. (Archivio Storico Lombardo)

RACrist. Rivista di Archeologia Cristiana 
RArqu. Revista de Arqueologia

RArt. Revue de l'Art

Rassd'A. Rassegna d'Arte

RassMon. Rassegna Monetaria

RAssyr. Revue d'Assyriologie

RASyr. Revue Archéologique Syrienne

RB. s. RBibl.

RBArch. Revue Belge d'Archéologie et d'Histoire de l'Art

RBibl. Revue Biblique

RBNum. Revue Belge de Numismatique

RBPhil. Revue Belge de Philologie et d'Histoire

RCC. Revue des Cours et Conférences

RCol. Rivista delle Colonie Italiane

RColIt. ersetzt durch RCol.

RCr. Revue Critique d'Histoire et de Littérature

RE. Paulys Realenzyklopädie der klassischen

Altertumswissenschaft. Neue Bearbeitung

REA. Revue des Études Anciennes

REG. Revue des Études Grecques

REgA. Revue de l'Égypte Ancienne

REHom. Revue des Études Homeriques

Reinach,RP. S. Reinach, Répertoire des Feintures Grecques et Romaines

Reinach,RR. S. Reinach, Répertoire des Reliefs Grecs et Romains

Reinach,RS. S. Reinach, Répertoire de la Statuaire Grecque et Romaine

Reinach,RV. S. Reinach, Répertoire des Vases Peints Grecs et Étrusques

REL. Revue des Études Latines

RendAccIt. Atti della Reale Accademia d'Italia. Rendiconti della Classe di Scienze Morali

RendAccNapoli Rendiconti della Reale Accademia di Archeologia, Lettere ed Arti

RendIstLomb. Reale Istituto Lombardo di Scienze e Lettere. Rendiconti

RendLinc. Atti dell' Accademia Pontificia dei Nuovi Lincei. Rendiconti della Reale Accademia dei Lincei

RendPontAcc. Atti della Pontificia Accademia Romana di Archeologia. Rendiconti

REV. ersetzt durch RV.

RevArtAncMod. Revue del'Art Ancien et Moderne

RevNum. Revue Numismatique

RevPhil. Revue de Philologie, de Littérature et d'Histoire Anciennes

RGG. Die Religion in Geschichte und Gegenwart

RGKBI. Römisch-Germanisches Korrespondenzblatt

RGVV. Religionsgeschichtliche Vorarbeiten und Versuche

RH. Revue Historique

RHitt. Revue Hittite et Asianique

RhM. Rheinisches Museum für Philologie

RHRel. Annales du Musée Guimet. Revue de l'Histoire des Religions
RIA. Rivista del Reale Istituto di Archeologia e Storia dell'Arte

Richter, Bronzes Metr. Mus. New York, Metropolitan Museum. G. M. A. Richter, Greek, Etruscan and Roman Bronzes

Richter, Cat. of Gems Metr. Mus. New York, Metropolitan Museum. G. M. A. Richter, Catalogue of Engraved Gems of the Classical Stylo

Richter, Handbook Metr. Mus. New York, Metropolitan Museum. G. M. A . Richter, Handbook of the Classical Collection

Richter-Hall G. M. A. Richter, Red-Figured Athenian Vases in the Metropolitan Museum of Art. With ... Drawings by L. F. Hall

RicRel. Ricerche Religiose

de Ridder, Bronzes Acrop. A. de Ridder, Catalogue des Bronzes trouvés sur l'Acropole d' Athènes

de Ridder, Bronzes Ant. Louvre Musées Nationaux du Louvre. A. de Ridder, Les Bronzes Antiques

de Ridder, Cat. Sommaire Louvre Musées Nationaux du Louvre. A. de Ridder, Catalogue Sommaire des Bijoux Antiques

de Ridder, Vas. de la Bilbl. Nat. A. de Ridder, Catalogue des Vases Peints de la Bibliothèque Nationale

RivCol. ersetzt durch RCol.

Rivlili. Rivista di Filologia e di Istruzione Classica

RivIGI. Rivista Indo-Greco-Italica di Filologia, Lingua, Antichità

RivStudLig. Rivista di Studi Liguri

RLV. s. RV.

RM. Mitteilungen des Deutschen Archäologischen Instituts. Römische Abteilung

Robert,SR. C. Robert, Die antiken SarkophagReliefs

Rodenwaldt,KdA. G. Rodenwaldt, Die Kunst der Antike

Roscher,ML. s. ML.

Roulez, Vas. de Leide J. Roulez, Choix de Vases Peints du Musée d'Antiquités de Leide

RPGR, ersetzt durch Reinach,RP.

RQu. Römische Quartalschrift für christliche Altertumskunde und für Kirchengeschichte

RScRel. Revue des Sciences Religieuses

RStOr. Rivista degli Studi Orientali

RStP. Rivista di Studi Pompeiani

RV. Reallexikon der Vorgeschichte, hrsg. von M. Ebert

v. Sacken, Sculpt. Wien E. v. Sacken, Die antiken Sculpturen des K. K. Münz- und AntikenCabinetes beschrieben und erklärt

v. Sacken-Kenner E.v. Sacken - F. Kenner, Die Sammlungen des K. K. Münz- und AntikenCabinetes, beschrieben 
SBBerl. Sitzungsberichte der Akademie der Wissenschaften zu Berlin. Phil.-hist. Klasse

SBHeid. Sitzungsberichte der Heidelberger Akademie der Wissenschaften. Phil.-hist. Klasse

SBLeipz. Berichte über die Verhandlungen der Sächsischen Akademie der Wissenschaften zu Leipzig. Phil.-hist. Klasse

SBLpz. ersetzt durch SBLeipz.

SBMünch. Sitzungsberichte der Alkademie der Wissenschaften zu München. Phil.-hist. Klasse

SBWien Sitzungsberichte der Akademie der Wissenschaften in Wien. Phil.-hist. Klasse

Schede, Meisterwerke Konstantinopel Meisterwerke der türkischen Museen zu Konstantinopel I. M. Schede, Griechische und römische Skulpturen des Antikenmuseums

v. Schneider, Album Wien R. v. Schneider, Album auserlesener Gegenstände der Antikensammlung des Allerhöchsten Kaiserhauses

Schrader, Marmorbildwerke der Akrop. Die archaischen Marmorbildwerke der Akropolis. Hrsg. von H. Schrader.

SchwNumR. Schweizerische Numismatische Rundschau

Sieveking, Slg. Loeb J. Sieveking, Die Terrakotten der Sammlung Loeb

Sieveking-Hackl J. Sieveking - R. Hackl, Die Königl. Vasensammlung zu München

SKond. Seminarium Kondakovianum

Smith, Sculpt. of the Parthenon, Brit. Mus. London, British Museum. A. H. Smith, The Sculptures of the Parthenon

Sogliano A. Sogliano, Le Pitture Murali Campane scoverte negli Anni I867-79

Soobšč. Kratkie Soobščenija v Dokladach i Polevych Issledovanijach Instituta Istorii Material'noj Kultury

SOsl. Symbolae Osloenses

SovArch. Sovetskaja Archeologija

Springer-Wolters A. Springer, Die Kunst des Altertums. Neu bearb. von A. Michaelis und P. Wolters

Staïs, Guide V. Staïs, Guide illustré. Marbres et Bronzes du Musée National (Athen)

StdZ. Stimmen der Zeit

SteMat. IStudi e Materiali di Storia delle Religioni

Stephani, Vasenslg. Ermitage [L. Stephani], Die Vasensammlung der Kaiserl. Ermitage

Stephanos Wiegand Stephanos Theodor Wiegand zum 6o. Geburtstag von Freunden und Verehrern dargebracht

StEtr. Studi Etruschi

StIt. Studi Italiani di Filologia Classica

Stuart Jones H. Stuart Jones, The Sculptures of the Palazzo dei Conservatori
Swed. Cypr. Exped. The Swedish Cyprus Expedition. Finds and Results of the Excavations in Cyprus I927-I93I

Sybel, Kat. Athen I. v. Sybel, Katalog der Sculpturen zu Athen

TfK. Tidskrift för Konstvetenskap

Thes. Lat. Thesaurus Linguae Latinae

Thieme-Becker Allgemeines Lexikon der bildenden Künstler. Hrsg. von U. Thieme und F. Becker

ThLZ, Theologische Literaturzeitung

Thouvenot, Cat. Madrid R. Thouvenot, Catalogue des Figurines et Objets de Bronze du Musée Archéologique de Madrid

Tillaeg til Billedtavler Ny Carlsberg Glyptotek. Tillaeg til Billedtavler af Antike Kunstvaerker

Tillaeg til Kat. F. Poulsen, Tillaeg til Katalog over Ny Carlsberg Glyptoteks Antike Kunstvaerker

Tillyard, Hope Vases E. M. IV.Tillyard, The Hope Vases

Tischbein, Hamilton Vases W. Tischbein, Collection of Engravings from Ancient Vases ... now in the Possession of Sir W. Hamilton

TrAPhAss. Transactions and Proceedings of the American Philological Association

TrBristol Transactions of the Bristol and Gloucestershire Archaeological Society

TrCumb. Transactions of the Cumberland and Westmorland Antiquarian and Archaeological Society

TrEssex Transactions of the Essex Archaeological Society

TrZ. Trierer Zeitschrift

TürkTarDerg. Türk Tarih, Arkeologya ve Etnografya Dergisi

UuF. Aus Unterricht und Forschung

VDI. Vestnik Drevnej Istorii

VuG. Vergangenheit und Gegenwart

WaG. Die Welt als Geschichte

Waldhauer, Ermitage Die antiken Skulpturen der Ermitage. Hrsg. von $O$. Waldhauer

Walter, Akropolismus. O. Walter, Beschreibung der Reliefs im kleinen Akropolismuseum in Athen

Watzinger, Vas. Tübingen C. Watzinger, Griechische Vasen in Tübingen

WBll. Wiener Blätter für die Freunde der Antiko

Welt d. Griechen, Kat. Heidelberg Die Welt der Griechen im Bilde der Originale der Heidelberger Universitätssammlung. Hrsg. von B. Neutsch

Wiad. Wiadomości Archeologicane

WJh. Wiener Jahreshefte

WiltshMag. Wiltshire Archaeological and Natural History Magazine 
Winter,KiB. Kunstgeschichte in Bildern. Abt. I: F. Winter, Das Altertum

Winter,Typen Die antiken Terrakotten (hrsg. von R. Kekulé). III F. Winter, Die Typen der figürlichen Terrakotten

WklPh. Wochenschrift für klassische Philologie

Wolters, Führer Glyptothek München P. Wolters, Führer durch die Glyptothek König Ludwigs I, zu München

WPZ. Wiener Prähistorische Zeitschrift

WSt. Wiener Studien

WüJbb. Würzburger Jahrbücher für die Altertumswissenschaft

WuS. Wörter und Sachen

IVV. O. Benndorf, Wiener Vorlegeblätter für archaeologische UUbungen

WVDOG. Wissenschaftliche Veröffentlichungen der Deutschen Orient-Gesellschaft

WZMorg. Wiener Zeitschrift für die Kunde des Morgenlandes

YaleCISt. Yale Classical Studies

Year'sW. The Year's Work in Classical Studies

YorkshAJ. Yorkshire Archaeological Journal
ZA. s. ZAssyr.

ZAesth. Zeitschrift für Aesthetik und allgemeine Kunstwissenschaft

ZAK. s. ZSchwAlt.

ZAssyr. Zeitschrift für Assyriologie

ZatW. Zeitschrift für die alttestamentliche Wissenschaft

ZBK. Zeitschrift für Bildende Kunst

ZDMG. Zeitschrift der Deutschen Morgenländischen Gesellschaft

ZDPV. Zeitschrift des Deutschen Palästina-Vereins

ZfE. Zeitschrift für Ethnologie

ZfK. Zeitschrift für Kunstgeschichte

ZKG. Zeitschrift für Kirchengeschichte

ZntW. Zeitschrift für neutestamentliche Wissenschaft

ZNum. Zeitschrift für Numismatik

Zpravy Zpravy Ceskoslovenskeho Statniho Archeologickeho Ustavu

ZSav. Zeitschrift der Savignystiftung für Rechtsgeschichte

ZSchwAlt. Zeitschrift für schweizerische Archaeologie und Kunstgeschichte

Sonstige Abkürzungen:

Bl. Blatt Bll. Blätter Bol. Boletin Jb. Jahrbuch Jbb. Jahrbücher Rev. Review, Revue
Boll. Bollettino

Jber. Jahresbericht

Riv. Rivista
Bull. Bulletin J. Journal Rdsch. Rundschau 